\title{
Caracterização dos candidatos ao uso de próteses auditivas em um serviço de saúde auditiva regional do estado do Rio Grande do Sul
}

\author{
Dayane Domeneghini Didoné ${ }^{1}$ Fernanda Freitas Vellozo² Michele Vargas Garcia ${ }^{3}$ Elenir Fedosse ${ }^{4}$
}

\begin{abstract}
RESUMO
Introdução - o Sistema único de saúde(SUS) disponibiliza uma portaria de saúde auditiva voltada a concessão de próteses auditivas. Essa portaria visa a reabilitação de sujeitos nas diferentes faixas etárias em todos os tipos e graus de perda auditiva. Objetivo - Caracterizar os usuários do Setor de Próteses auditivas de um Centro de Especialidades e de Diagnóstico da região central do estado do Rio Grande do Sul. Metodologia - Trata-se de uma pesquisa quantitativa, retrospectiva e transversal . A população alvo desta pesquisa foi constituída por todos os candidatos ao uso de próteses auditivas no período de 2000 a 2010. Resultados - Predominou o grupo de adultos e idosos, com ensino fundamental incompleto, que recebem até dois salários mínimos. As profissões "aposentado" e "do lar" predominaram na amostra. Conclusão - As principais características dos indivíduos deste estudo foram: adultos e idosos, com ensino fundamental incompleto, aposentados ou do lar, que recebem até dois salários mínimos.
\end{abstract}

Descritores: Perda Auditiva; Sistema Único de Saúde; Aparelhos Auditivos.

\section{Characterization of candidates for the use of hearing aids in a health service regional hearing the state of Rio Grande do Sul}

\begin{abstract}
Introduction - The National Health Care System (SUS) provides a concierge hearing care focused on providing hearing aids. This ordinance seeks rehabilitation of subjects in different age groups in all types and degrees of hearing loss. Objective - To characterize users Sector Hearing a Specialty Center and Diagnostics central region of Rio Grande do Sul. Material and Method - This is a quantitative, retrospective and documentary. The target population of this research consisted of all candidates for the use of hearing aids in the period 2000-2010. Results - The predominant group of adults and seniors with elementary school, receiving up to two minimum wages. The professions "retired" and "home" predominated in the sample. Conclusion - The main characteristics of the subjects in this study were adults and seniors with elementary school, retired or housewives, they receive up to two minimum wages.
\end{abstract}

Descriptors: Hearing loss; Unified Health System; Hearing Aids.

\footnotetext{
${ }^{1}$ Mestre em Distúrbios da comunicação humana pela Universidade Federal de Santa Maria (UFSM), Santa Maria, RS, Brasil.

${ }^{2}$ Graduada em Fonoaudiologia pela Universidade Federal de Santa Maria (UFSM), Santa Maria, RS, Brasil.

${ }^{3}$ Doutora em Distúrbios da comunicação humana pela Universidade Federal de São Paulo (UNIFESP), São Paulo, SP, Brasil.

${ }^{4}$ Doutora em Linguística pela Universidade Estadual de Campinas (UNICAMP), Campinas, SP, Brasil.
} 


\section{Introdução}

De acordo com a Organização Mundial de Saúde, $10 \%$ da população mundial tem algum déficit auditivo. No Brasil, calcula-se que 15 milhões de homens e mulheres tenham algum tipo de perda auditiva (neurossensorial, condutiva ou mista) IBGE'1.

A perda neurossensorial origina-se na orelha interna ou ao longo das vias neurais; o dano geralmente ocorre na cóclea, sendo que suas células ciliadas são danificadas a ponto de não conseguirem transmitir os impulsos elétricos ao cérebro. Tais lesões são usualmente permanentes, levando ao déficit sensorial e à distorção dos sons. A perda auditiva condutiva é uma perda limitada a patologias de orelha externa e média. Já a perda auditiva mista se refere ao acontecimento simultâneo de lesões na orelha externa/média e da orelha interna².

A deficiência auditiva (DA) pode ser diagnosticada em diferentes faixas etárias. Para o diagnóstico precoce da DA foi instituída como lei em 2010 a triagem auditiva neonatal (TAN). Existem diferentes indicadores de risco para a deficiência auditiva infantil, segundo 0 Joint ComitteonInfantHearing ${ }^{3}$, tais como a permanência em Unidade de Terapia Intensiva Neonatal (UTIN) por 48 horas ou mais, antecedente familiar de perda auditiva ou consanguinidade, infecções congênitas, ventilação mecânica prolongada e síndromes.

Nos adultos e idosos existem diversas etiologias para a perda de audição, entre elas a presbiacusia, a qual é definida como um declínio da função auditiva devido ao processo de envelhecimento, sendo associada com a deterioração de outros sistemas sensoriais. A deficiência auditiva pode ocorrer associada ao declínio cognitivo, demência, falta de destreza manual e acuidade visual ${ }^{4}$.

As consequências desses déficits auditivos podem ser amenizadas com o uso das próteses auditivas, as quais fornecem amplificação dos sons ambientais e sons da fala, minimizando os prejuízos da perda de audição e melhorando a qualidade de vida dos usuários. As próteses auditivas são recomendadas para pessoas com perdas auditivas neurossensoriais e também para as que apresentam déficits de condução que não podem ser submetidos a procedimentos cirúrgicos ${ }^{5}$.

Com o uso das próteses auditivas espera-se que o usuário melhore a capacidade de percepção dos sons e compreensão de fala, uma vez que a amplificação sonora proporcionada pelas mesmas reintroduz a estimulação de sons que 0 indivíduo não ouvia naturalmente 6 .

Em 2004, considerando as precárias condições de acesso da população brasileira aos procedimentos de saúde auditiva, o Ministério da Saúde instituiu a Política Nacional de Atenção à Saúde Auditiva, por meio da Portaria $n^{0} 2.073$ de 28 de setembro, que garante desde o diagnóstico da deficiência auditiva à protetização dos usuários, ou seja, indicação e adaptação de próteses auditivas, pelo Sistema Único de Saúde (SUS) 7 .

Segundo esta portaria, um Programa Nacional de Saúde Auditiva é importante pela necessidade de estabelecer normas de atuação em nível nacional na área da prevenção, diagnóstico e re/habilitação precoces da deficiência auditiva.

No Rio Grande do Sul, a instituição do programa de saúde auditiva aconteceu em 2006 em um município de médio porte, sendo que deste então foi estabelecida parceria com uma instituição de nível superior.

Esse estudo teve por objetivo caracterizar os candidatos ao uso de próteses auditivas de um Centro de Especialidades e de Diagnóstico de referência regional do Rio Grande do sul.

\section{Metodologia}

Esta pesquisa é de abordagem quantitativa, do tipo retrospectivo, documental e transversal, realizada a partir da consulta aos arquivos/prontuários dos candidatos ao uso de próteses auditivas do Programa de Saúde Auditiva.

Este estudo está vinculado ao projeto de pesquisa "Caracterização dos Usuários de um Serviço de Referência Municipal e Regional em Especialidades de Média Densidade Tecnológica" registrado no Comitê de Ética em Pesquisa de uma instituição pública sob os números 0091.0.243.000-11.

O estudo abrange o período de 2000 a 2010 sendo que a coleta de dados ocorreu entre maio e julho do ano de 2011, totalizando a pesquisa a 841 prontuários. A faixa etária dos indivíduos variou de 1 a 99 anos, sendo divididas e classificadas da seguinte forma: criança: zero a 12 anos, adolescentes: 13 a 21 anos, adultos: 22 a 64 anos e idosos a partir de 65 anos.

As variáveis analisadas nesse estudo dizem respeito à idade, sexo, escolaridade, profissão e renda per capta, por tratar-se de uma caracterização dos candidatos do programa. 
Foram incluídos na amostra todos os candidatos à protetização que tiveram indicação de próteses auditivas no dado período. Nenhum sujeito foi excluído da amostra.

Após o levantamento dos dados os resultados foram dispostos em planilhas, utilizando o programa Microsoft Excel e a análise utilizada foi com o teste Qui-quadrado.

\section{Resultados}

Foi observada que a quantidade de homens que aguardavam próteses auditivas por concessão do programa de saúde auditiva foi maior do que a quantidade de mulheres, porém não houve diferença estatisticamente significante.

Em relação à faixa etária, os grupos de idosos e adultos mostraram-se estatisticamente elevados quando comparados com a faixa etária de adolescentes e crianças.

Sobre a escolaridade, predominou o grupo de indivíduos com ensino fundamental incompleto.

As profissões "aposentado" e "do lar" foram as que predominaram na amostra, sendo essa diferença estatisticamente significante quando comparadas com outras profissões.

Quanto ao salário, o rendimento de até 2 salários mínimos predominou de forma estatisticamente significante na amostra.

Tabela 1 - Distribuição absoluta e relativa para o sexo, faixa etária, escolaridade,profissão e renda; e nível mínimo de significância para o teste Qui-quadrado de homogeneidade para comparação de proporções.

\begin{tabular}{|c|c|c|c|}
\hline \multirow[t]{2}{*}{ Variáveis } & \multicolumn{3}{|c|}{ Total amostra $(n=841)$} \\
\hline & $\mathrm{n}$ & $\%{ }^{*}$ & $\mathrm{p} \S$ \\
\hline \multicolumn{4}{|l|}{ Sexo } \\
\hline Feminino & 402 & 47,8 & \multirow[t]{2}{*}{0,202} \\
\hline Masculino & 439 & 52,2 & \\
\hline \multicolumn{4}{|l|}{ Faixa etária } \\
\hline Criança & 48 & 5,7 & \multirow{4}{*}{$<0,001$} \\
\hline Adolescente & 38 & 4,5 & \\
\hline Adulto & 213 & 25,5 & \\
\hline Idoso & 537 & 64,2 & \\
\hline \multicolumn{4}{|l|}{ Escolaridade } \\
\hline Fundamental incompleto & 456 & 62,7 & \multirow{7}{*}{$<0,001$} \\
\hline Fundamental completo & 106 & 14,6 & \\
\hline Médio incompleto & 16 & 2,2 & \\
\hline Médio completo & 88 & 12,1 & \\
\hline Ensino superior & 27 & 3,7 & \\
\hline Analfabeto & 18 & 2,5 & \\
\hline Alfabetizado & 16 & 2,2 & \\
\hline \multicolumn{4}{|l|}{ Profissão } \\
\hline Outros & 153 & 20,0 & \multirow{7}{*}{$<0,001$} \\
\hline Agricultor & 31 & 4,1 & \\
\hline Aposentado & 300 & 39,3 & \\
\hline Do lar & 160 & 20,9 & \\
\hline Estudante & 70 & 9,2 & \\
\hline Motorista & 20 & 2,6 & \\
\hline Servente de Obras & 30 & 3,9 & \\
\hline \multicolumn{4}{|l|}{ Renda } \\
\hline Até 2 salários & 790 & 97,9 & \multirow{3}{*}{$<0,001$} \\
\hline Até 4 salários (Mais de 2 a 4) & 13 & 1,6 & \\
\hline Até 6 salários (Mais de 4 a 6) & 4 & 0,5 & \\
\hline
\end{tabular}

§: Qui quadrado de homogeneidade 


\section{Discussão}

A perda auditiva afeta milhões de brasileiros, desde crianças até idosos, e programas de saúde pública que visam o diagnóstico e reabilitação desses indivíduos são fundamentais para proporcionar desenvolvimento de habilidades linguísticas, cognitivas e sociais, assim como evitar o declínio dessas funções.

O programa de saúde auditiva da presente pesquisa atende candidatos residentes na cidade sede do programa e de outros 30 municípios que compõe a $4^{\text {a }}$ Coordenadoria Regional de Saúde do Rio Grande do Sul.

Nesse estudo, a quantidade de indivíduos idosos $(64,2 \%)$ que procuraram atendimento para reabilitação da deficiência auditiva foi maior quando comparada $\mathrm{E}$ com outras faixas etárias. Esses dados concordam parcialmente com outro estudo $^{8}$, onde a porcentagem de indivíduos idosos protetizados foi de $45 \%$. Nos Estados Unidos ${ }^{9}$ cerca de $30 \%$ das pessoas com mais de 65 anos, e $50 \%$ das pessoas com mais de 75 anos apresentam perda auditiva.

O constante crescimento da população idosa com presbiacusia justifica a elevada procura por serviços de reabilitação auditiva. Segundo dados do Instituto Brasileiro de Geografia e Estatística (IBGE)1, no ano de 2000 a população geral do Brasil era de 171.279.882 habitantes, sendo 13.915.357(8,1\%) de idosos. Para o ano de 2020, a estimativa da população geral é de 219.077.729, sendo a de idosos 28.321 .801 (12,9\%). Nesse estudo também foi verificado um grande número de idosos, constatando essa procura pelos serviços de reabilitação auditiva.

Estudos brasileiros ${ }^{10}$ apontam para uma porcentagem de $63,93 \%$ de idosos com perda auditiva em uma população de 43.610 habitantes. Se comparada essa proporção com a de recém-nascidos que apresentam perda auditiva (cerca de 3 crianças em cada 1000 triadas $^{11}$ ), percebe-se que a porcentagem de perda auditiva em idosos é maior, o que vai ao encontro dos resultados desse estudo.

Ressalta-se que, referente à profissão, prevaleceu o grupo de aposentados. Esse resultado é justificado pelo fato da maioria dos candidatos ao uso de próteses auditivas ser idosos, sendo que é direito constitucional no Brasil que os trabalhadores urbanos do sexo masculino se aposentem a partir dos 65 anos, do sexo feminino a partir dos 60 anos de idade, e os trabalhadores rurais com cinco anos a menos ${ }^{12}$.

Nesta pesquisa houve predominância do sexo masculino, embora sem diferença estatisticamente significante. Amaioria dos indivíduos era idosa e acredita-se que essa variável pode estar relacionada aos achados deste estudo, o que foi de encontro à literatura da área. Os estudos atuais com a população idosa indicam predominância do sexo feminino ${ }^{13,14}$, considerando que a partir de 65 anos a presbiacusia é mais prevalente em mulheres e até os 65 anos em homens. Nesta amostra a população idosa predominante foi a de mais de 65 anos, o que discorda dos estudos apresentados, já que a predominância de sexo foi masculino. P6, p7. Essa discordância pode ser em decorrência da distribuição dos indivíduos por faixa etária, sendo a predominante a de idosos com mais de 65 anos.

Apesar da maior prevalência da perda auditiva em homens ressalta-se a necessidade de investimentos voltados à prevenção em toda a população idosa sem distinção de sexo, já que, além da presbiacusia, outros fatores podem potencializar a perda auditiva, como alterações metabólicas e vasculares, exposição ao ruído, medicamentos, alterações nutricionais e estresse, os quais podem ser minimizados por meio de medidas preventivas.

Em relação à escolaridade, o ensino fundamental incompleto foi o que predominou entre os indivíduos, associada à prevalência da população idosa, a qual predominou nesse estudo. Estudos sobre as condições de ensino de idosos no Brasil| ${ }^{15}$ também referem baixa escolaridade nessa população, sendo que grande parte desses indivíduos não concluem 0 ensino fundamental. Em se tratando de saúde, o nível educacional interfere diretamente na capacidade de entendimento de tratamentos prescritos e na prática do autocuidado, o que no caso de perdas auditivas, pode refletir no entendimento, uso e cuidados com as próteses auditivas ${ }^{16}$.

Quanto a renda per capta, a maioria dos sujeitos apresentou renda de até dois salários mínimos, corroborando os achados de outro estudo ${ }^{17}$, no qual prevaleceu, na amostra estudada, idosos com renda de um a três salários mínimos. Esses dados podem ser explicados pelo baixo nível de escolaridade em ambos os estudos, o que potencializa os riscos maiores de adoecimento dessas populações, já que o acesso ao tratamento e a prática do cuidado podem estar comprometidas nestes casos.

A partir da caracterização dos candidatos ao uso de próteses auditivas E26 é possível conhecer as diferentes realidades regionais, a fim de proporcionar um melhor atendimento a essa população, desde a prevenção até a reabilitação auditiva. 


\section{Conclusão}

A caracterização dos candidatos ao uso de próteses auditivas de um Centro de Especialidades e de Diagnóstico de referência regional do Rio Grande do sul demonstrou que à espera para concessão de próteses auditivas tem-se mais idosos, homens, aposentados, com ensino fundamental incompleto, que ganham até dois salários mínimos.

\section{Referências bibliográficas}

1. IBGE - Instituto Brasileiro de Geografia e Estatística. Censo Demográfico, 2000.

2. Bevilacqua MC, Martinez MAN, Balen SA, Pupo AC, Reis ACM, Frota S. Tratado de Audiologia. São Paulo: Santos, 2011. 3. Joint Committee on Infant Hearing $(\mathrm{JClH})$. Year 2007 Position statement: principles and guidelines for early hearing detection and intervention mprograms. Pediatrics. 2007;120(4):898-921.

4. Kiessling J, Pichora-Fuller MK, Gatehouse S, Stephens D, Arlinger S,Chisolm T, et al. Candidature for and delivery of audiological services: special needs of older people. Int J Audiol. 2003;42 (2):92-101.

5. Gasparin M, Menegotto $\mathrm{HH}$, Cunha CS. Psychometric properties of the international outcome inventory for hearing aids. Braz J Otorhinolaryngol. 2010;76(1):85-90.

6. Santos SN, Petry T, Costa MJ. Índice percentual de reconhecimento de sentenças no silêncio e no ruído: efeitos da aclimatização no indivíduo avaliado sem as próteses auditivas. Rev. CEFAC. 2010; 12(5):733-740.

7. OMS - Organização Mundial da Saúde. Política Nacional de Atenção à Saúde Auditiva, 2004.

8. Viera AF, Menegotto IH, Teixeira AR, Millão LF. Presença de deficiência auditiva e hipertensão em adultos e idosos. RBCEH. 2009; 6(2):245-253.

9. Neves VT, Feitosa MAG. Envelhecimento do processamento temporal auditivo. Psic.: Teor. e Pesq. 2002;18(3):275-82. 10. Meneses C, Mário MP,Marchori LMM, Melo JJ, Freitas ERFS. Prevalência de perda auditiva e fatores associados na população idosa de Londrina, Paraná: Estudo Preliminar. CEFAC. 2010; 12(3).

11. Grupo de Apoio a Triagem Auditiva Neonatal Universal (GATANU).

12. BRASIL. Constituição (1988). Constituição da República Federativa do Brasil. Brasília, DF, Senado, 1998.

13. Cruz MS, Lima MCP, Santos LF, Duarte YAO, Lebrão ML, Ramos - Cerqueira ATA. Deficiência auditiva referida por idosos no Município de São Paulo, Brasil: prevalência fatores associados (Estudo SABE, 2006). Cad. Saúde Pública. 2012; 28(8):1479-1492.

14. Mattos LC, Veras RP. A prevalência da perda auditiva em uma população de idosos da cidade do Rio de Janeiro: um estudo seccional. Rev Bras Otorrinolaringol. 2007; 73(5):654:59.

15. Anderson MIP. Saúde e Condições de vida do idoso no Brasil. Textos Envelhecimento. 1998; 1(1).

16. Meireles VC, Matsuda, LM, Coimbra JAH, Mathias TAF. Características dos idosos em área de abrangência do programa saúde da família na região Noroeste do Paraná: contribuições para a gestão do cuidado em enfermagem. Saúde e Sociedade. 2007; 16(1): 69-80.

17. Paiva KM, Cesar CLG, Alves MCGP, Barros MBA, Caradina L, Goldbaum M. Envelhecimento e deficiência auditiva referida: um estudo de base populacional. Cad. Saúde Pública. 2011; 27(7):1292-1300.

\section{Dayane Domeneghini Didoné}

Endereço para correspondência - Av. Roraima, n 1000, Hospital Universitário de Santa Maria - setor de Fonoaudiologia. Bairro Camobi, CEP 97105-900, Santa Maria, RS, Brasil.

E-mail: dayanedidone@yahoo.com.br

Lattes: http://lattes.cnpq.br/3125014661196452

Fernanda Freitas Vellozo - fevellozo@yahoo.com.br

Michele Vargas Garcia - michelemvg@outlook.com

Elenir Fedosse-efedosse@gmail.com

Enviado em 26 de março de 2014.

Publicado em 07 de julho de 2014. 
